\title{
PHASE-SPACE FINITE ELEMENT METHODS APPLIED TO THE FIRST-ORDER FORM OF THE TRANSPORT EQUATION
}

\author{
William R. Martin, Carl E. Yehnert, Leonard Lorence and James J. Duderstadt \\ Department of Nuclear Engineering, The University of Michigan, Ann Arbor, Michigan, U.S.A.
}

\begin{abstract}
The application of the finite element method to the first-order form of the neutron transport equation is reviewed. The general theoretical foundation of the finite element application is summarized, including a derivation of the weak form, a discussion of the treatment of all boundary conditions as natural boundary conditions and a few remarks concerning convergence. Results of the 1-D application are presented including a description of the discontinuous phase-space finite elements. The 2-D application is discussed and its application to the classic ray effect problem is examined. It is concluded that the finite element method does alleviate the ray effect but at the considerable expense of computational time and memory requirements. To address this concern, a new 'segmentation' scheme for the 2-D application is described. This scheme yields satisfactory results for the ray effect problem while reducing the computational cost by nearly an order of magnitude. Finally a few remarks are presented concerning the time-dependent application and the paper concludes with some general comments concerning the overall application of the finite element method to the first-order equation and comparison with alternative methods.
\end{abstract}

\section{INTRODUCTION}

The finite element method is the name commonly applied to the expansion of the solution to a set of partial differential equations in a set of local basis functions. Either variational or weighted residual methods are then used to arrive at a sparse matrix representation of the original problem. This method has received considerable attention over the past decade as a promising alternative to conventional techniques such as spherical harmonics or discrete ordinates for solving the transport equation.

The finite element method was first applied in the mid-1950s to a variety of problems in structural mechanics. Since that time finite element methods have been developed for such diverse areas as fluid mechanics, heat transfer and neutron diffusion. The success of the finite element method may be partially attributed to its versatility for treating quite general classes of partial differential equations. However, it also presents decided advantages for handling complex, irregular geometries, and its firm theoretical foundation in approximation theory guarantees the convergence of the approximate solution in most cases of interest.

The application of the finite element method to transport problems has been a relatively recent venture. A variety of approaches have been studied, such as finite element expansions in both the space and angle variables for both the traditional (non-self-adjoint) and the even- and odd-parity second-order forms of the transport equation. These methods may be described as phase-space finite element methods as opposed to the hybrid scheme, wherein finite element techniques are used for the spatial variables and conventional discrete ordinates are used for the angular variables. In general, none of the phase-space finite element efforts have resulted in a 'production-level' finite element transport code to rival discrete ordinates codes or the combined finite element-discrete ordinates codes; however, research in this area is continuing at a rapid pace. Of particular note is the extensive research directed at applying phase-space finite element methods to the second-order form of the transport equation by a number of research groups.

In this paper, our attention will be directed at phasespace finite element methods for transport problems based on the first order (conventional) form of the transport equation. We begin with a brief discussion of the theoretical formulation of the method, including error and convergence rate estimates. We review past efforts on applications to both one- and twodimensional problems, including a summary of results comparing computational efficiency and numerical accuracy of these methods when compared to other approaches. We also review the effort associated with a more recent 'segmented' algorithm that holds promise for improving computer run times and storage requirements for multidimensional problems. As will be noted in the following discussion, the issue of 
computational efficiency is a critical one for the eventual success of finite element methods for solving the first-order transport equation. A brief discussion of the solution of time-dependent problems will also be given. Finally we will conclude with several remarks concerning the comparison between the first- and second-order form finite element methods and more conventional approaches based on discrete ordinates.

\section{THEORETICAL FOUNDATIONS}

\subsection{General background}

The basic procedure involved in applying finite element methods is quite simple. First the problem domain including the boundary is partitioned into a number of non-overlapping adjacent cells, called 'finite elements'. The solution is expanded in a finite set of basis functions defined on each individual cell in terms of a set of parameters (the expansion coefficients) associated with the nodes, which may be discrete points on the element boundaries or points within the element domain. Generally the nodal parameters are chosen to be the value of the solution and/or its derivatives at the node. Adjacent elements are then related to each other through the nodal parameters, a fact which serves to enforce continuity conditions across element boundaries. Choosing the basis functions to be polynomials defined on local regions consisting of only a few finite elements results in a piecewise polynomial expansion of the solution. There are several options for developing the set of simultaneous algebraic equations to determine the expansion coefficients. Once these have been obtained, standard techniques can be employed to solve the matrix equation for the expansion coefficients. This method offers such a variety of approaches, starting with the shapes of the finite elements, to the form chosen for the trial functions, to the method selected for setting up and solving the matrix equation, that a wide number of science and engineering applications can be considered.

Mathematically, the finite element method corresponds to choosing an appropriate approximation subspace and expanding the unknown solution in a set of basis functions that span the approximating subspace. It is theoretically demonstrable for many situations that the finite element solution is as 'close' to the actual solution as any other member of the approximating subspace. It is in fact the projection of the actual solution onto the approximating subspace. By choosing successively large subspaces (i.e. refining the mesh) one can guarantee convergence of the finite element solutions to the actual solution.

The finite element method can be implemented either via variational or weighted residual techniques. For self-adjoint problems, it is attractive to implement the method by minimizing an appropriate functional (most commonly the Ritz or Roussopoulos) using trial functions defined on the finite element subspace. Requiring the functional to be stationary results in a matrix equation for the expansion coefficients. A second approach is the class of weighted residual methods in which an integral form of the original equation is developed. In the most common form known as the Galerkin method, weight functions are chosen that correspond to the basis functions defining the approximate solution. By using the local basis functions, both methods yield sparse matrix systems which are often banded in such a way that computational and storage advantages can be realized.

For the case of a self-adjoint system such as the onespeed diffusion equation, both variational and weighted residual methods are equivalent. However since the first-order form of the transport equation is not self-adjoint, there is no available extremum variational principle. We have no choice but to apply the finite element method to the solution of the weak or integral law form of this equation.

We will now briefly review the theoretical formulation of the finite element method as applied to the first-order transport equation. This review will include a description of the transport equation, the derivation of its equivalent weak or integral law form, comments on the treatment of boundary conditions and some remarks concerning error estimates and convergence rates. This section is based to a large extent on the earlier work of Ukai (1972) and subsequent works by Martin (1976) and Martin and Duderstadt (1977).

\subsection{Integral law (weak) form of the transport equation}

Let us first consider the derivation of the integral law corresponding to the first-order transport equation, assuming time-independence and the one-speed approximation

$$
\begin{aligned}
\hat{\mathbf{\Omega}} \cdot \nabla Q(r, \hat{\mathbf{\Omega}}) & +\Sigma_{t}(r) Q(r, \mathbf{\Omega}) \\
= & \int_{4 \pi} \mathrm{d} \mathbf{\Omega}^{\prime} \Sigma_{s}\left(r, \boldsymbol{\Omega}^{\prime} \cdot \mathbf{\Omega}\right) Q\left(r, \mathbf{\Omega}^{\prime}\right)+S(r, \boldsymbol{\Omega})
\end{aligned}
$$

subject to a specified angular flux on the incoming boundary

$$
Q\left(r_{\mathrm{s}}, \mathbf{\Omega}\right)=Q_{0}\left(r_{\mathrm{s}}, \mathbf{\Omega}\right), \mathbf{\Omega} \cdot \hat{\mathbf{e}}_{\mathrm{s}}<\mathbf{0}
$$

where $r_{\mathrm{s}}$ is a point on the boundary. We introduce the following notation for convenience:

$$
\begin{gathered}
R=\text { spatial domain } \\
4 \pi=\text { angular domain }
\end{gathered}
$$




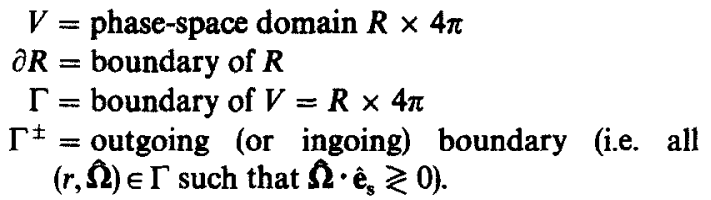

We will also need to introduce the space of allowable solutions (the 'energy' space)

$$
H_{\mathrm{E}}=\left\{\operatorname{Real} \psi\left(r, \hat{\mathbf{\Omega})} \mid \iint_{V} \mathrm{~d} r \mathrm{~d} \hat{\mathbf{\Omega}}\left[|\psi|^{2}+|\nabla \psi|^{2}\right]<\infty\right\}\right.
$$

and the following inner products and norms associated with $H_{\mathrm{E}}$ :

$$
\begin{gathered}
(f, g) \equiv \iint_{V} \mathrm{~d} r \mathrm{~d} \mathbf{\Omega} f(r, \hat{\mathbf{\Omega}}) g(r, \hat{\mathbf{\Omega}}) \\
\langle f, g\rangle \equiv \iint_{\Gamma} \mathrm{d} S \mathrm{~d} \hat{\mathbf{\Omega}} \mathbf{\Omega} \cdot \dot{\mathbf{e}}_{\mathrm{s}} f\left(r_{\mathrm{s}}, \hat{\mathbf{\Omega}}\right) g\left(r_{\mathrm{s}}, \hat{\mathbf{\Omega}}\right) \\
\langle f, g\rangle \pm \equiv \iint_{\Gamma^{*}} \mathrm{~d} S \mathrm{~d} \hat{\mathbf{\Omega}}\left|\hat{\mathbf{\Omega}} \cdot \mathbf{e}_{\mathrm{s}}\right| f\left(r_{\mathrm{s}}, \hat{\mathbf{\Omega}}\right) g\left(r_{\mathrm{s}}, \hat{\mathbf{\Omega}}\right) \\
\|f\|_{0} \equiv(f, f)^{1 / 2} \\
\|f\|_{1} \equiv[(f, f)+(\nabla f, \nabla f)]^{1 / 2} .
\end{gathered}
$$

The weak or integral law form of equation (1) is obtained by multiplying equation (1) by an arbitrary $\psi(r, \hat{\Omega}) \in H_{\mathrm{E}}$ and integrating over the phase-space domain $V$. An integration by parts then yields surface integrals into which the boundary condition equation (2) is included. The resultant weak or integral law formulation may then be stated:

Find a function $Q(r, \hat{\Omega}) \in H_{\mathrm{E}}$ such that for all $\psi(r, \Omega) \in H_{\mathrm{E}}$

$$
\begin{aligned}
-(Q, \mathbf{\Omega} \cdot \nabla \psi)+\langle Q, \psi\rangle_{+}+( & K Q, \psi) \\
& =(S, \psi)+\left\langle Q_{0}, \psi\right\rangle_{-}
\end{aligned}
$$

It is readily demonstrated that equation (8) and the original partial differential equation plus boundary conditions, equations (1) and (2), are equivalent.

\subsection{Boundary conditions}

The boundary condition $Q=Q_{0}$ on $\Gamma^{-}$is included in the integral law equation (8). It will be naturally satisfied by the solution $Q(r, \Omega)$, even though we did not explicitly require the entire solution space $H_{\mathrm{E}}$ to satisfy it. This is an example of a natural boundary condition. Such boundary conditions are not imposed directly on the space of trial functions, but rather result as a consequence of the integral law itself. If we had not employed an integration by parts, then we would have had to restrict our allowable space of trial functions to those $Q \in H_{\mathrm{E}}$ that also satisfied the boundary condition $Q=Q_{0}$ on $\Gamma^{-}$. This latter situation is known as essential boundary conditions, because they are imposed on the space of trial functions.

The derivation of natural boundary conditions using an integration by parts is a general feature of finite element methods. For the first-order transport equation, all boundary conditions may be treated as natural boundary conditions. That is, one simply substitutes the expression for $Q$ on $\Gamma^{-}$into the surface term

$$
\langle Q, \psi\rangle_{-}=\iint_{\Gamma^{-}} \mathrm{d} S \mathrm{~d} \hat{\mathbf{\Omega}}\left|\hat{\mathbf{\Omega}} \cdot \hat{\mathbf{e}}_{\mathrm{s}}\right| Q\left(r_{\mathrm{s}}, \hat{\mathbf{\Omega}}\right) \psi\left(r_{\mathrm{s}}, \hat{\mathbf{\Omega}}\right) .
$$

If the boundary conditions are implicit, one substitutes the relation between $Q$ on $\Gamma^{-}$to $Q$ on $\Gamma^{+}$into $\langle Q, \psi\rangle$. This feature will become more evident when we consider the specific case of one-dimensional plane symmetry in Section 3.

\subsection{The approximate integral law}

The integral law is now in a form that is amenable to approximation by way of the finite element method. That is, rather than attempting to find a solution $Q(r, \widehat{\Omega})$ of equation (8) in the space $H_{\mathrm{E}}$, we seek a solution $Q^{h}(r, \boldsymbol{\Omega}) \in S^{h}$ such that equation (8) is satisfied for all $\psi^{h}(r, \Omega) \in S^{h}$. Here $h$ is a parameter that depends on the mesh spacing to be used in the approximate solution and $S^{h}$ is a specially constructed subspace of $H_{\mathrm{E}}$ with basis functions $\psi_{i}^{h}(r, \hat{\Omega}), i=1,2, \ldots, N$, where $N$ is the dimension of $S^{h}$ (typically the number of nodes in the mesh). Here it is important to note that the basis functions are local in the sense that

$$
\iint_{V} \mathrm{~d} r \mathrm{~d} \hat{\mathbf{\Omega}} \psi_{i}^{h}(r, \hat{\mathbf{\Omega}}) \psi_{j}^{h}(r, \boldsymbol{\Omega})
$$

is non-zero only when the nodes $i$ and $j$ are close together.

When we restrict ourselves to this subspace, we arrive at an approximate form of the integral law: find $Q^{h}(r, \boldsymbol{\Omega}) \in S^{h}$ such that for all $\psi^{h}(r, \boldsymbol{\Omega}) \in S^{h}$,

$$
\begin{aligned}
-\left(Q^{h}, \hat{\mathbf{\Omega}} \cdot \nabla \psi^{h}\right)+\left\langle Q^{h}, \psi^{h}\right\rangle_{+} & +\left(K Q^{h}, \psi^{h}\right) \\
& =\left(S, \psi^{h}\right)+\left\langle Q_{0}, \psi^{h}\right\rangle .
\end{aligned}
$$

Since $S^{h}$ is finite dimensional and $Q^{h} \in S^{h}$, we can expand $Q^{h}$ in the basis functions for $S^{h}$,

$$
Q^{h}(r, \mathbf{\Omega})=\sum_{j=1}^{N} \phi_{j} \psi_{j}^{h}(r, \boldsymbol{\Omega})
$$


and it is sufficient to require equation (10) to hold for all $\psi_{i}^{h}(r, \hat{\Omega}), i=1,2, \ldots, N$ to ensure that equation (10) is valid for all $\psi^{h}(r, \widehat{\Omega}) \in S^{h}$ :

$$
\begin{aligned}
& -\left(\sum_{j=1}^{N} \phi_{j} \psi_{j}^{h}, \hat{\mathbf{\Omega}} \cdot \nabla \psi_{i}^{h}\right)+\left\langle\sum_{j=1}^{N} \phi_{j} \psi_{j}^{h}, \psi_{i}^{h}\right\rangle_{+} \\
& +\left(K \sum_{j=1}^{N} \phi_{j} \psi_{j}^{h}, \psi_{i}^{h}\right)=\left(S, \psi_{i}^{h}\right)+\left\langle Q_{0}, \psi_{i}^{h}\right\rangle_{-}
\end{aligned}
$$

If we take the summation outside the integrals, we arrive at the matrix system

$$
\mathbb{A} \underline{\phi}=\underline{S}
$$

where

$$
\begin{aligned}
A_{i j}= & -\left(\psi_{j}^{h}, \hat{\mathbf{\Omega}} \cdot \nabla \psi_{i}^{h}\right)+\left\langle\psi_{j}^{h}, \psi_{i}^{h}\right\rangle \\
& +\left(K \psi_{j}^{h}, \psi_{i}^{h}\right) \\
S_{i}= & \left(S, \psi_{i}^{h}\right) \\
\underline{\phi}= & \operatorname{col}\left(\phi_{1}, \phi_{2}, \ldots, \phi_{N}\right)
\end{aligned}
$$

where the inner product notation of equation (3) has been utilized.

At this point we might note that the construction of the subspace $S^{\text {h }}$ has not entered the discussion, and equation (10) could very well be applicable to any Galerkin formulation. However, the choice of a finite element basis considerably simplifies the calculation. More importantly, this choice results in convergence to the actual solution as the mesh spacing is refined $(h \rightarrow 0)$. That is, if $Q(r, \Omega)$ is the actual solution, then

$$
\lim _{h \rightarrow 0} Q^{h}(r, \hat{\mathbf{\Omega}}) \rightarrow Q(r, \hat{\mathbf{\Omega}})
$$

(in some suitable measure or norm).

\subsection{Convergence of the finite element solution}

In general, if the finite element method is applied to a second-order, self-adjoint operator, it can be shown (Strang and Fix, 1973) that the $\mathrm{L}_{2}$ error $\varepsilon$ in the approximate solution $Q^{h}(r)$,

$$
\varepsilon \equiv\left[\int \mathrm{d}^{3} r\left|Q(r)-Q^{h}(r)\right|^{2}\right]^{1 / 2}
$$

is less than the $L_{2}$ error for any other approximation that can be formed within the finite element subspace $S^{h}$

$$
\begin{gathered}
{\left[\int \mathrm{d}^{3} r\left|Q(r)-Q^{h}(r)\right|^{2}\right]^{1 / 2} \leqslant\left[\int \mathrm{d}^{3} r\left|Q(r)-\chi^{h}(r)\right|^{2}\right]^{1 / 2}(14)} \\
\text { for all } \chi^{h} \in S^{h} .
\end{gathered}
$$

But the finite element subspaces of most interest consist of piecewise polynomials. Therefore we can appeal to approximation theory to ascertain the degree to which the space of piecewise polynomials $S^{h}$ can approximate an arbitrary function $f(r)$. In particular, if $f(r)$ has a square-integrable gradient over the domain $R$, there exists an approximation $f^{h}(r) \in S^{h}$ such that

$$
\left[\int \mathrm{d}^{3} r\left|f(r)-f^{h}(r)\right|^{2}\right]^{1 / 2} \leqslant C h^{k+1}
$$

where $h$ is the mesh spacing, $k$ the degree of the piecewise polynomials, and $C$ is a constant independent of $h$ or $k$. For example, $k=1$ for linear piecewise polynomials, and therefore for this case

$$
\left[\int \mathrm{d}^{3} r\left|f(r)-f^{h}(r)\right|^{2}\right]^{1 / 2} \leqslant \mathrm{Ch}^{2}
$$

But from equation (14) we know that $Q^{h}(r)$ is at least as close to the true solution $Q(r)$ as this particular approximation. Therefore

$$
\left[\int \mathrm{d}^{3} r\left|Q(r)-Q^{h}(r)\right|^{2}\right]^{1 / 2} \leqslant \mathrm{Ch}^{k+1}
$$

and as $h \rightarrow 0$, we conclude that $Q^{h}(r) \rightarrow Q(r)$ (in the $\mathrm{L}_{2}$ measure) with $O\left(h^{k+1}\right)$ convergence.

The above remarks, however, do not apply to the first-order transport equation. Ukai (1972) has proven a weaker estimate for the $L_{2}$ error in the approximate solution,

$$
\left\|Q-Q^{h}\right\|_{0} \leqslant\left\|Q-\chi^{h}\right\|_{1}
$$

for arbitrary $\chi^{h} \in S^{h}$. From approximation theory we know there exists a $\chi^{h} \in S^{h}$ such that

$$
\left\|Q-\chi^{h}\right\|_{1} \leqslant \mathrm{Ch}^{k}
$$

which immediately yields the error estimate

$$
\left\|Q-Q^{h}\right\|_{0} \leqslant \mathrm{Ch}^{k}
$$

which is $O(h)$ less than the corresponding error estimate given above in equation (17) for the second-order, selfadjoint case. However, numerical results obtained by Martin (1975) substantiate the following convergence for the error in the approximate solution of this equation

$$
\left[\iint_{V} d^{3} r d \hat{\mathbf{\Omega}}\left|Q(r, \hat{\mathbf{\Omega}})-Q^{h}(r, \hat{\mathbf{\Omega}})\right|^{2}\right]^{1 / 2} \leqslant C h^{k+1} .
$$

Therefore the finite element method yields the 'best' possible solution from the space of allowable trial functions. Since approximation theory tells us that the space of piecewise polynomials is capable of approximating the unknown solution to any degree of accuracy, we are assured that the finite element solution will be at least as accurate. 


\section{ONE-DIMENSIONAL PLANE SYMMETRY}

\subsection{Derivation of the finite element equations}

To illustrate the finite element approach, we will consider the one-dimensional transport equation in plane geometry. For simplicity, we will consider the problem of a homogeneous slab on the interval $0 \leqslant x \leqslant 1$

$$
\begin{aligned}
u \frac{\partial Q}{\partial x} & +\Sigma_{t}(x) Q(x, \mu)=\sum_{l=0}^{L}\left(\frac{2 l+1}{2}\right) \\
& \times \Sigma_{\mathrm{sl}}(x) P_{l}(\mu) \int_{-1}^{1} \mathrm{~d} \mu^{\prime} P_{l}\left(\mu^{\prime}\right) Q\left(x, \mu^{\prime}\right)+S(x, \mu)
\end{aligned}
$$

subject to reflecting(symmetry) boundary conditions at $x=0$,

$$
Q(0, \mu)=Q(0,-\mu)
$$

and a specified incoming flux at $x=1$,

$$
Q(1, \mu)=Q_{0}(\mu), \mu<0
$$

In this case our space $H_{\mathrm{E}}$ becomes

$$
H_{\mathrm{E}}=\left\{\psi(x, \mu) \mid \int_{0}^{1} \mathrm{~d} x \int_{-1}^{1} \mathrm{~d} \mu\left[\psi^{2}+\left|\frac{\partial \psi}{\partial x}\right|^{2}\right]<\infty\right.
$$

and once again we choose a subspace $S^{h} c H_{\mathrm{E}}$ and expand the solution to the 1-D analog of the approximate integral law, equation (10), in terms of the basis functions of $S^{\text {h }}$ :

$$
Q^{h}(x, \mu)=\sum_{j=1}^{N} \phi_{j} \psi_{j}^{h}(x, \mu)
$$

Substituting equation (26) into the 1-D approximate integral law then yields the following system of albegraic equations

$$
\sum_{j=1}^{N} A_{i j} \phi_{j}=S_{i}, \quad i=1,2, \ldots, N
$$

where

$$
\begin{aligned}
A_{i j}= & -\int_{0}^{1} \mathrm{~d} x \int_{-1}^{1} \mathrm{~d} \mu \mu \psi_{j}^{h}(x, \mu) \frac{\partial \psi_{i}^{h}}{\partial x} \\
& +\int_{0}^{1} \mathrm{~d} \mu \mu \psi_{i}^{h}(1, \mu) \psi_{j}^{h}(1 \mu) \\
& -\int_{-1}^{0} \mathrm{~d} \mu \mu \psi_{i}^{h}(0, \mu) \psi_{j}^{h}(0, \mu) \\
& +\int_{-1}^{0} \mathrm{~d} \mu \mu \psi_{i}^{h}(0,-\mu) \psi_{j}^{h}(0, \mu) \\
& +\int_{0}^{1} \mathrm{~d} x \int_{-1}^{1} \mathrm{~d} \mu \Sigma_{t}(x) \psi_{i}^{h}(x, \mu) \psi_{j}^{h}(x, \mu)
\end{aligned}
$$

$$
\begin{aligned}
& -\sum_{l=0}^{L}\left(\frac{2 l+1}{2}\right) \int_{0}^{1} \mathrm{~d} x \Sigma_{s l}(x) \int_{-1}^{1} \mathrm{~d} \mu \\
& \times \psi_{i}^{h}(x, \mu) P_{l}(\mu) \int_{-1}^{1} \mathrm{~d} \mu^{\prime} \psi_{j}^{h}\left(x, \mu^{\prime}\right) P_{l}\left(\mu^{\prime}\right) .
\end{aligned}
$$

Therefore the finite element method has led to a system of algebraic equations that can be solved by standard methods for the expansion coefficients $\phi_{i}$.

The detailed steps leading from equation (22) to equation (27) are similar to the general case discussed above and may be found in Martin and Duderstadt (1977). It should be noted however that the inclusion of the reflecting boundary condition into the integral law was performed in the direction of neutron motion by eliminating the incoming flux in favor of the outgoing flux:

$$
Q(0, \mu)=Q(0,-\mu), \quad \mu>0 .
$$

It has been observed (Martin and Duderstadt, 1977) that eliminating in the other direction yields completely erroneous results and it is important to 'follow the neutrons', which is a basic tenet of discrete ordinates methods.

\subsection{Construction of the finite element subspace}

To illustrate how to construct a suitable finite element subspace $S^{h}$, we provide specific details for onedimensional plane geometry. In general, the finite element method is employed in a nodal fashion. That is, the expansion coefficients for the solution are nodal parameters that are typically the value of the solution or one of its derivatives at a node of the space-angle mesh. It is common to use Lagrange interpolation polynomials over each local mesh or finite element. The values of the solution at the nodes are the expansion coefficients, hence the unknowns in the system of equations. We could also employ Hermite interpolation polynomials on a given finite element mesh; however, in this case each node would include additional parameters representing the values of both the solution and its derivatives. Lagrange finite element schemes typically result in continuity of the solution everywhere in the mesh, although derivatives are generally discontinuous across interelement boundaries. Hermitian schemes typically result in continuity of one or more partial derivatives everywhere in the mesh.

Since the transport equation is only of first order, one can expect at most continuity of the angular flux. Even this may be too restrictive for the angular variable. Therefore the use of Lagrangian elements that preserve continuity in the solution but not its derivatives would appear to be a proper choice for transport problems. 
Lagrangian elements may be determined for a specific element type or formulated for multidimensional elements in terms of direct products of two or more onedimensional Lagrangian basis functions.

The more general method is to shape the basis functions to the particular element. For example, a triangular element with three nodes can uniquely represent a linear polynomial over a two-dimensional surface, with each node contributing a piece of data. That is, a general linear polynomial in two variables $p(x, y)=a_{0}+a_{1} x+a_{2} y$ requires the value of three parameters to be uniquely determined. If the values of the solution at the nodes of a triangle are taken to be the three parameters, the linear polynomial is uniquely determined within the triangle. With Lagrangian elements, where the nodal parameters are the value of the unknown solution, a convenient basis for a triangle consists of three linear polynomials, each of which is unity at one node and zero at the other nodes. This will result in the expansion coefficients being identical to the value of the solution at the nodes. This concept of choosing a basis consisting of functions that are unity at a particular node and zero at all other nodes is typical of Lagrangian elements, and it is used for the direct product basis functions in our following discussion.

For multidimensional elements one can also formulate basis functions that are direct products of simple one-dimensional basis functions. For example, the one-dimensional plane geometry basis functions could be written as the product of space and angle functions

$$
\psi(x, \mu)=\psi_{x}(x) \psi_{u}(\mu) .
$$

A convenient consequence of the direct product basis functions is that the components of the matrix elements $A_{i j}$ are separable into products of spatial and angular integrals. For example, the contribution to $A_{i j}$ in equation (27) due to the streaming term becomes

$$
\begin{aligned}
\int_{0}^{1} \mathrm{~d} x \int_{-1}^{1} \mathrm{~d} \mu \mu \psi_{j} \frac{\partial \psi_{i}}{\partial x} & =\int_{0}^{1} \mathrm{~d} x \frac{\mathrm{d} \psi_{i x}(x)}{\mathrm{d} x} \psi_{j x}(x) \\
& \times \int_{-1}^{1} \mathrm{~d} \mu \mu \psi_{i \mu}(\mu) \psi_{j \mu}(\mu)
\end{aligned}
$$

where $i x$ is the $x$ node corresponding to node $i$. This greatly simplifies the calculation of the integrals because the spatial and angular integrals may be performed independent of one another.

Although the ranges of integration of the integrals in $A$ are given over the full spatial and angular ranges, in fact the local definition of the basis functions greatly reduces the range of integration, and the components of $A_{i j}$ generally vanish if nodes $i$ and $j$ are not adjacent (for linear elements). There is one notable exception, however. The scattering contribution to $A$

$$
\begin{aligned}
\sum_{i=0}^{L}\left(\frac{2 l+1}{2}\right) & \int_{0}^{1} \mathrm{~d} x \Sigma_{s i} \psi_{i x} \psi_{j x} \\
& \times \int_{-1}^{1} \mathrm{~d} \mu P_{l}(\mu) \psi_{i \mu} \int_{-1}^{1} \mathrm{~d} \mu^{\prime} P_{l}\left(\mu^{\prime}\right) \psi_{j \mu}\left(\mu^{\prime}\right)
\end{aligned}
$$

is a product of integrals of basis functions rather than an integral of a product of basis functions. Therefore scattering couples all the angular nodes (although the spatial coupling is unaffected). This would be expected on physical grounds since there is a finite probability of scattering from one direction to another.

One can define higher order basis functions, either as a higher-order polynomial over a general element such as a triangle or as a direct product of higher-order onedimensional polynomials. To construct higher-order elements, one introduces additional nodes (e.g. one additional node for quadratic basis functions) and defines the higher-order polynomials over several nodes (vs two nodes for linear elements). This results in the coupling of nodes that would not be coupled by linear elements. This is not a concern with angular elements because all nodes are coupled by scattering in any event. But this increased coupling does present severe problems if higher-order elements are used in space.

Although the matrix elements are relatively simple to compute analytically, the sheer number of integrals for even moderate sized problems forces one to simplify the work even further. For example, all integrals can be performed on the standard interval $[-1,+1]$ by Gaussian quadrature, then mapped into the particular mesh interval by a simple linear transformation.

\subsection{Discontinuous basis functions}

In plane geometry it is well known that the transport equation may have discontinuities in the angular flux at $\mu=0$, at interfaces, or at boundaries. We can handle this discontinuity by generalizing the finite element method to include discontinuous basis functions. Continuity in the angular variable is not required during the derivation of the integral law in Section 3.1, since the transport equation in plane geometry has no angular derivatives. Therefore the use of discontinuous angular elements is simply a matter of constructing basis functions that are discontinuous at $\mu=0$ (for plane geometry) and being careful to evaluate the integral in a piecewise fashion. This is easily accomplished by splitting the basis function at $\mu=0$ into two basis functions, one for $\mu=0^{-}$and the other for $\mu=0^{+}$. Thus there is a double node at $\mu=0$. For a multidimensional problem where two angles are 
needed, one can use direct product basis functions of these discontinuous one-dimensional functions.

The situation for the spatial domain is somewhat different, since the exact solution to the transport equation must be continuous. Yet even here there may be situations at which the solution exhibits a near discontinuity. For example, the simple problem of a strong source of neutrons in a strong absorber surrounded by a vacuum will result in an angular flux with nearly discontinuous spatial dependence at the vacuum boundaries. To more adequately approximate this behavior using finite element methods, one can proceed as with the angular variable and simply construct discontinuous basis functions at the desired spatial positions, thus allowing the approximate solution to be discontinuous. However, the presence of the spatial derivative in the streaming term necessitates care when the transport equation is integrated over the spatial domain. That is, the integration across the discontinuity will yield surface terms which must be carefully incorporated into the integral law.

The basic idea, the details of which may be found in (Martin and Duderstadt, 1977) is to split up the integral over the physical domain into integrals over domains which are separated by the assumed discontinuities. This is done prior to the integration by parts, which to be valid requires continuity of the flux within the range of integration. Integrating over the separate domains by parts then yields interface terms at the discontinuities. These interface terms are then treated in a fashion similar to the treatment of the reflecting boundary conditions discussed above-continuity of the angular flux is imposed in the direction of neutron motion at the interface,

$$
\begin{array}{ll}
Q\left(X_{\mathrm{D}}^{+}, \mu\right)=Q\left(X_{\mathrm{D}}^{-}, \mu\right), & \mu>0 \\
Q\left(X_{\mathrm{D}}^{-}, \mu\right)=Q\left(X_{\mathbf{D}}^{+}, \mu\right), & \mu<0
\end{array}
$$

where we have assumed that $X_{\mathrm{D}}$ is the discontinuity. The result is a matrix element $I_{i j}$

$$
\begin{aligned}
I_{i j}= & -\int_{-1}^{0} \mathrm{~d} \mu \mu \psi_{j}^{h}\left(X_{\mathrm{D}}^{+}, \mu\right) \\
& \times\left[\psi_{i}^{h}\left(X_{\mathrm{D}}^{+}, \mu\right)-\psi_{i}^{h}\left(X_{\mathrm{D}}, \mu\right)\right] \\
& -\int_{0}^{1} \mathrm{~d} \mu \mu \psi_{j}^{h}\left(X_{\mathrm{D}}^{-}, \mu\right) \\
& \times\left[\psi_{i}^{h}\left(X_{\mathrm{D}}^{+}, \mu\right)-\psi_{i}^{h}\left(X_{\mathrm{D}}^{-}, \mu\right)\right]
\end{aligned}
$$

which is additive to the earlier matrix element $A_{i j}$ defined in equation (27). As with the imposition of reflecting boundary conditions, we have observed that imposing continuity of the angular flux in the direction opposite to neutron motion yields erroneous results.
Since the space of trial functions $S^{h}$ is no longer continuous at the specified spatial discontinuity, but continuity in the direction of particle travel is imposed within the integral law, the net result is that continuity of the angular flux in the direction of particle motion appears as a natural interface boundary condition. Although there was no need to mention it earlier, our earlier formulation treated continuity of the angular flux as an essential boundary condition because continuity was imposed on the space $S^{h}$ by construction of the basis functions. Therefore, the discontinuous basis function scheme allows more flexibility for the approximate solution to match the actual solution.

\subsection{Numerical results}

Numerical results for the 1-D plane geometry application have been previously reported (Martin, 1975; Martin, 1976; Martin and Duderstadt, 1977) and are summarized below.

1. The convergence rate (as measured by the $\mathrm{L}_{2}$ error) for the source problem has been shown on the basis of numerical experiments to be $O\left(h^{k+1}\right)$ where $h$ is the mesh area and $k$ the degree of the finite element. To the authors' knowledge this rate has yet to be proven theoretically.

2. Eigenvalue convergence rates for linear elements appear to be $O\left(h^{3}\right)$, which appears to be consistent with the $O\left(h^{2}\right)$ rate observed for the flux error. The use of discontinuous angular elements at $\mu=0$ is essential in order to obtain this convergence rate in that the use of continuous elements resulted in a marked loss in the convergence rate.

3. For 1-D eigenvalue problems, the finite element method is competitive with conventional discrete ordinates codes for accuracy and computational efficiency.

4. The prediction of criticality eigenvalues for anisotropic scattering slabs has been performed and the results indicate that the finite element method works quite well, even with extremely thin slabs.

5. For problems characterized by extreme heterogeneities, such as the well-known four region slab problem devised by Reed (1971), the finite element method yields excellent results and substantiates the use of discontinuous spatial elements for problems with strong material discontinuities.

\section{TWO-DIMENSIONAL RECTANGULAR TRANSPORT}

The application of phase-space finite element methods to the 2-D first-order transport equation has been investigated by Yehnert (1978). The following 
discussion summarizes the results of that effort, including conclusions concerning the accuracy and efficiency of the finite element method as well as its ability to mitigate the ray effect.

\subsection{Derivation of the finite element equations}

Phase-space finite elements can be readily extended to two-dimensional domains. For the purpose of this discussion, neutron transport in the rectangular coordinate system of Fig. 1 will be treated, where the assumption of translational symmetry in the $z$ direction has been exploited to yield a two-dimensional problem. We will also assume, without loss of generality, symmetry in the azimuthal angle about $\varphi=\pi$. The angular domain is chosen as $\mu=\cos (\theta)=$ $[-1,1]$ and $\varphi=[0, \pi]$. The first-order neutron transport equation in this coordinate system becomes :

$$
\begin{aligned}
\mu \frac{\partial Q}{\partial x}+ & \sqrt{1-\mu^{2}} \cos \varphi \frac{\partial Q}{\partial y}+\Sigma_{l}(x, y) Q(x, y, \mu, \varphi) \\
= & S(x, y, \mu, \varphi)+\sum_{l=0}^{L} \frac{2 l+1}{2 \pi} \Sigma_{\mathrm{sl}}(x, y) \int_{-1}^{1} \mathrm{~d} \mu^{\prime}(x) \\
& \quad \times(x) \int_{0}^{\pi} \mathrm{d} \varphi^{\prime} \sum_{k=0}^{l} Y_{l}^{k}\left(\mu^{\prime}, \varphi^{\prime}\right) Y_{l}^{k}(\mu, \varphi) Q\left(x, y, \mu^{\prime}, \varphi^{\prime}\right)
\end{aligned}
$$

+ boundary conditions.

The integral law is obtained in the manner discussed in Section 2.2 above by application of the inner product $(, \psi)$ to equation (33) where $\psi$ is a member of $H_{\mathrm{E}}(V)$. Integration by parts converts the streaming operator term to:

$$
\begin{aligned}
& -\int \mathrm{d} V \mu Q(x, y, \mu, \varphi) \frac{\partial \psi}{\partial x}+2 \int_{0}^{b} \mathrm{~d} y \int_{0}^{1} \mathrm{~d} \mu \int_{0}^{\pi} \mathrm{d} \varphi Q(a, y, \mu, \varphi) \psi(a, y, \mu, \varphi) \\
& -2 \int_{0}^{b} \mathrm{~d} y \int_{-1}^{0} \mathrm{~d} \mu \mu \int_{0}^{\pi} \mathrm{d} \varphi Q(0, y, \mu, \varphi) \psi(0, y, \mu, \varphi)-\int \mathrm{d} V \sqrt{1-\mu^{2}} \cos \varphi Q(x, y, \mu, \varphi) \frac{\partial \psi}{\partial y} \\
& +2 \int_{0}^{a} \mathrm{~d} x \int_{-1}^{1} \mathrm{~d} \mu \sqrt{1-\mu^{2}} \int_{0}^{\pi / 2} \mathrm{~d} \varphi \cos \varphi Q(x, b, \mu, \varphi) \psi(x, b, \mu, \varphi)-2 \int_{0}^{a} \mathrm{~d} x \int_{-1}^{1} \\
& \mathrm{~d} \mu \sqrt{1-\mu^{2}} \int_{\pi / 2}^{\pi} \mathrm{d} \varphi \cos \varphi \psi(x, 0) Q(x, 0)
\end{aligned}
$$

where the spatial domain is the rectangular region of Fig. 2. Other terms of the integral law similarly follow.

The Galerkin weighted residual method follows the expansion of the angular flux in terms of functions that constitute a basis for a subset of $H_{\mathrm{E}}(V)$ :

$$
Q(x, y, \mu, \varphi)=\sum_{j=1}^{N} \phi_{j} \psi_{j}(x, y, \mu, \varphi) .
$$

The set of simultaneous equations for $\phi_{j}$ are obtained by allowing the arbitrary function $\psi(V)$ to assume the identities of members of $\left\{\psi_{j}\right\}$ :

$$
\sum_{j=1}^{N} A_{i j} \phi_{j}=S_{i}, \quad i=1,2, \ldots, N .
$$

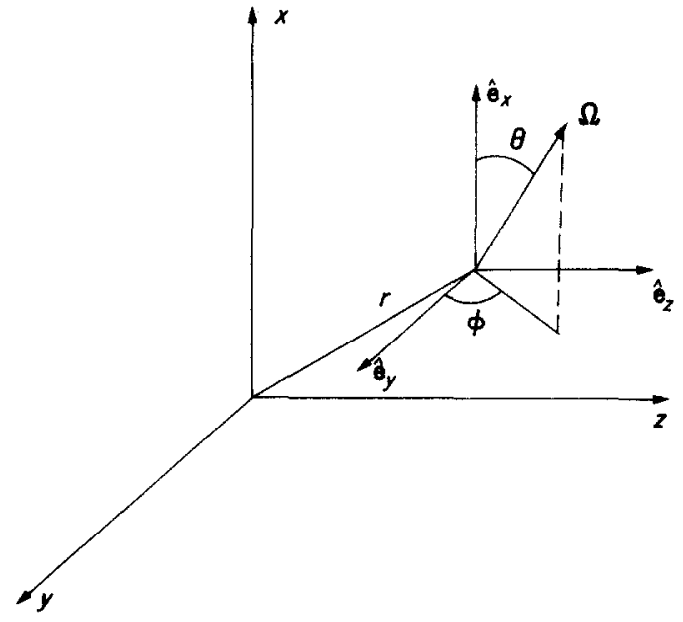

Fig. 1. Coordinate system for 2-D geometry.

\subsection{Construction of finite element subspace}

The set of basis functions are chosen as finite element functions constructed on the discretized domain of the problem. By choosing Lagrange basis functions, the unknowns in equation (36) are the approximate angular flux at the nodes of the discretized domain. In this study, the subspace was formed from the direct product of rectangular polynomials in space and angle. The rectangular bilinear and biquadratic polynomials are: bilinear: $\quad b_{1}+b_{2} x+b_{3} y+b_{4} x y$

biquadratic: $c_{1}+c_{2} x+c_{3} y+c_{4} x y+c_{5} x^{2}$

$$
+c_{6} y^{2}+c_{7} x y^{2}+c_{8} x^{2} y+c_{9} x^{2} y^{2} .
$$

These rectangular polynomials are themselves constructed from the tensor product of one-dimensional polynomials.

Discontinuous angular elements may be constructed by placing additional nodes on the grid in those directions corresponding to $\boldsymbol{\Omega} \cdot \hat{\mathbf{e}}_{\mathbf{s}}=\mathbf{0}$. For the physical domain of Fig. 2 , these directions are $\varphi=\pi / 2$ at the $\mathrm{Cl}$ and $\mathrm{C} 3$ edges, and $\mu=0$ at edges $\mathrm{C} 2$ and $\mathrm{C} 4$. 


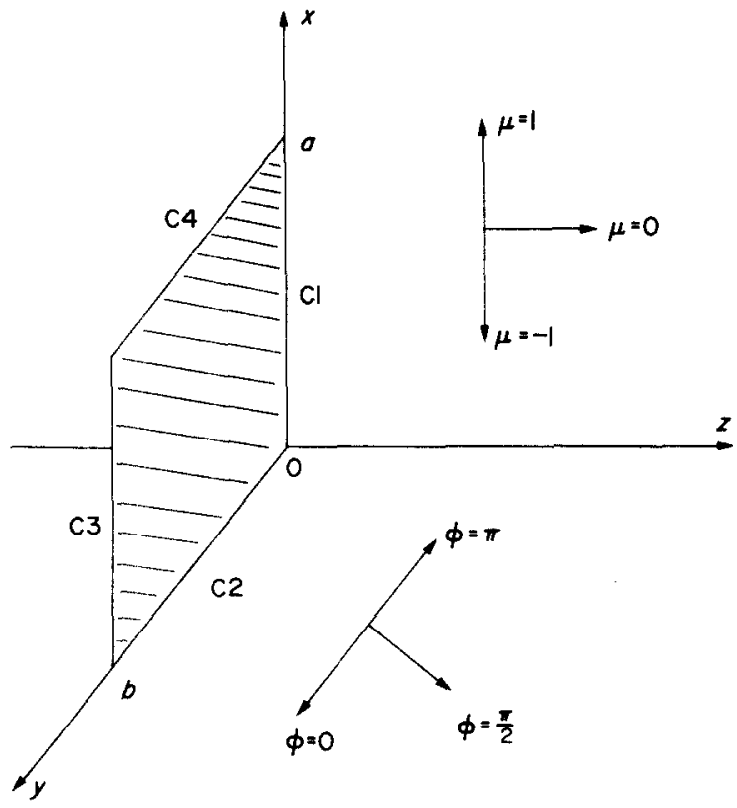

Fig. 2. Physical domain for 2-D geometry.

\subsection{Construction of the coefficient matrix and source vector}

The coefficient matrix is assembled by performing the phase-space integration over the streaming, scattering and removal terms. Since the basis functions are local, the only non-zero terms are those whose basis functions have overlapping support. The sparse system that results can, with proper indexing of nodes, be converted into a block-banded matrix. Each block in this structure represents the interaction between nodes of the $y, \mu$ and $\varphi$ directions. The block bandwidth of the coefficient matrix is $2 I_{x}+1$ where $I_{x}$ is the order of the basis functions in the $x$-direction.

The matrix is off-diagonally dominant and nonsymmetric due to the influence of the streaming and scattering terms. For instance, consider the evaluation of a typical streaming integral term :

$$
\int_{y_{i-1}}^{y_{i+1}} \mathrm{~d} y \psi_{i}(y) \frac{\mathrm{d} \psi_{i}}{\mathrm{~d} y} .
$$

For a uniform mesh spacing $h$, the derivative $\mathrm{d} \psi_{i} / \mathrm{d} y$ has, for linear basis functions, the value $1 / h$ in the mesh to the left of the node ${ }_{i}$, and $-1 / h$ in the mesh to the right of node ${ }_{i}$. In all diagonal terms, where $i=j$, this leads to an exact cancellation of the integrals over the two meshes $\left[y_{i-1}, y_{i}\right]$ and $\left[y_{i}, y_{i+1}\right]$. On the off-diagonal, the integration of equation (38) is performed over one mesh, either $\left[y_{i-1}, y_{i}\right]$ of $\left[y_{i}, y_{i+1}\right]$, so there is no cancellation. However, the opposite signs in $\mathrm{d} \psi / \mathrm{d} y$ leads to an antisymmetric matrix. Furthermore, the derivatives are proportional to $1 / h$, so that as $h \rightarrow 0$ the streaming terms lead to off-diagonal domination of the matrix.

The source vector for the matrix equation is also calculated by phase space integration. For instance, internal sources are approximated by an expansion in terms of the same set of basis functions used to represent the angular flux :

$$
S(x, y, \varphi)=\sum_{i=1}^{N} S_{i} \psi_{i}(x, y, \mu, \varphi) .
$$

The inner product of this term with the basis set yields its contribution to the source vector. Similarly, the incoming boundary flux term will consist of a line integral in space along the edge of the problem domain, plus an angular integration over all incoming angles associated with the corresponding spatial boundary.

Spectral reflection can be treated in the Galerkin finite element method with the aid of physical insight. The neutrons at a reflecting boundary which leave the system at a point $r$ in the direction $\Omega$ reappear at the same spatial point, but with a new direction $\hat{\Omega}^{\prime}$, which has the same angle with respect to the plane perpendicular to the reflecting boundary and passing through the original direction $\mathbf{\Omega}$. Fortunately in the coordinate system and rectangular region of Fig. 2 , only one of the angular variables changes along any one side of the boundary.

Consider the case of reflection on the line $x=0$. The boundary terms associated with the integration by parts of the streaming inner product term:

$$
\left(\mu \frac{\partial Q}{\partial x}, \psi\right)
$$

are:

$$
\begin{aligned}
& -2 \int_{0}^{\pi} \mathrm{d} \varphi \int_{-1}^{0} \mathrm{~d} \mu \mu \int_{0}^{b} \mathrm{~d} y Q(0, y, \mu, \varphi) \psi(0, y, \mu, \varphi) \\
& -2 \int_{0}^{\pi} \mathrm{d} \varphi \int_{0}^{1} \mathrm{~d} \mu \mu \int_{0}^{b} \mathrm{~d} y Q(0, y,-\mu, \varphi) \psi(0, y, \mu, \varphi) .
\end{aligned}
$$

Reflection is taken into account by changing $\mu \rightarrow-\mu$ in the second term of equation (41) to obtain:

$$
\begin{aligned}
-2 \int_{0}^{\pi} \mathrm{d} \varphi \int_{-1}^{0} \mathrm{~d} \mu \mu \int_{0}^{b} \mathrm{~d} y Q(0, y, \mu, \varphi) \\
\quad \times[\psi(0, y, \mu, \varphi)-\psi(0, y,-\mu, \varphi)] .
\end{aligned}
$$

The angular flux may now be expanded in a set of basis functions :

$$
Q(0, y, \mu, \varphi)=\sum_{i=1}^{n} \phi_{i} \psi_{i}(x, y, \mu, \varphi) .
$$


Thus reflection can be handled within the standard Galerkin finite element method provided the interaction between basis functions and the reflecting boundary is properly treated.

\subsection{Solution procedures}

Two different solution strategies were proposed for this matrix equation, block LU-decomposition and block-SOR. These block methods were chosen to minimize the storage requirements for the coefficient matrix. The computer code FTRAN2 (Yehnert, 1978) was developed to test the two numerical methods. The direct solution technique proved to be most accurate. As expected, decreased scattering and larger mesh size tended to improve the convergence of the block-SOR method.

Elimination methods such as block LUdecomposition have large storage requirements, even when most of the matrix is stored on disk file, and long execution times. The segmentation algorithm to be discussed in Section 5 can substantially reduce these penalties.

An artificial solution was constructed to test the capabilities of FTRAN2. This was done by finding an expression for the internal source for a specific functional form of the angular flux in the transport equation. These studies clearly demonstrated the expected improvement in solution accuracy as both the spatial and angular mesh were refined. Increased convergence to the analytical solution was also observed as the order of the angular finite elements was increased. The results indicated that the code is a bit more accurate at a specified incoming angular flux boundary than at a reflecting boundary. However, this difference is reduced with mesh refinement.

\subsection{Solution on a ray-effect domain}

The ray effect is a direct consequence of solving the neutron transport equation along discrete rays or directions, as in the discrete ordinates method. Briggs et al. (1975) have shown that the ray effect is mitigated by numerical approximations which transform the hyperbolic system with its accompanying 'characteristics' into an elliptical system with no characteristics. Since the second-order transport equation was employed in that study, it was possible to show analytically that the coefficients of the second-order terms yielded a characteristic polynomial equation which had no real roots and was thus elliptical.

The present application of the finite element method employs the first-order form of the transport equation, so a corresponding analytical study of the ray effect was not possible. However, some appeal can be made to physical arguments to illustrate how the ray effect is reduced in the finite element method.

In the Galerkin approach, the transport equation is weighted by trial functions having a piecewise polynomial shape on local regions in the phase-space, and then integrated over all space and angle. This process yields a matrix equation in which the source vector accounts for all sources in the phase-space through the interaction of the source interpolation function with the piecewise polynomial trial functions on each finite element. Also, the matrix itself accounts for all of the geometrical and material detail in phase space through the interaction of polynomials in the flux expansion with the trial functions on each finite element. There are no characteristic directions to integrate along, and no particular neutron paths to follow. The streaming operator is in a sense 'smeared' over a range of directions within an angular finite element instead of evaluated along one direction. This results in mitigation of the ray effect at some expense in accuracy, depending on how well the trial functions can approximate the angular dependence. We are always assured that by increasing the order of the basis polynomials, and by decreasing the mesh spacing of the finite elements, we can effect uniform convergence to the exact solution.

The ray effect has been studied for the classic problem shown in Fig. 3. The region consists of a material with uniform total cross section $\Sigma_{\mathrm{t}}=0.75$ and uniform scattering cross section $\Sigma_{s}$. The scattering ratio, defined as $c=\Sigma_{\mathrm{s}} / \Sigma_{\mathrm{t}}$ has been allowed to vary between 0 and 1 for the purposes of this study. The material contains a square flat source in one corner of the region. As shown, both reflecting and vacuum boundary conditions are used.

Studies on this domain indicated that refinement of the angular mesh was necessary to eliminate the ray

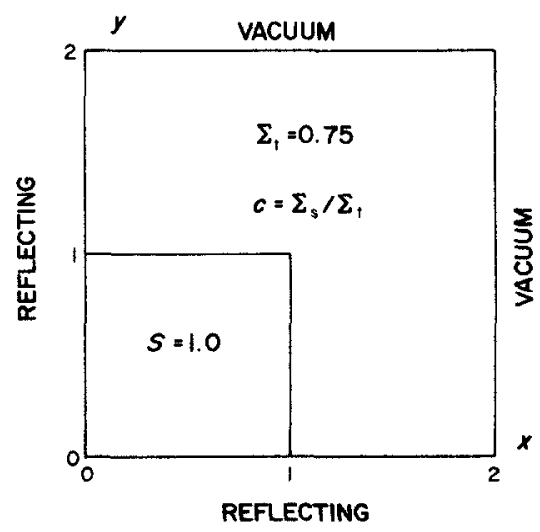

Fig. 3. Domain for ray-effect problem. 
effect. It was found that the shape of the angular flux is badly misrepresented when too few angular nodes are used to interpolate it, especially at the edge of a source or strong absorber where it may be rapidly varying. The integration of the angular flux produces a distorted scalar flux tantamount to a ray effect. Minimal refinement of the angular domain (from a $3 \times 3$ rectangular mesh to a $5 \times 5$ ) provided a reasonable representation for the angular flux.

On the refined angular mesh, the scalar flux profiles were in good agreement with comparable FENT (Miller et al., 1973) calculations. FENT is a twodimensional phase-space finite element code based on the second-order transport equation. Results from discrete ordinates codes such as TWOTRAN-II (Lathrop and Brinkley, 1973) and TRIDENT (Seed $e t$ al., 1977) were markedly inferior even with ray-effect mitigating options employed. The scalar fiux profiles obtained with these four codes are compared in Fig. 4. FTRAN2 was run on a $7 \times 7$ spatial mesh using biquadratic angular elements. A finer spatial mesh was used in the other calculations.

With respect to the other three codes, the time and storage required to eliminate the ray effect using FTRAN2 were unreasonable. If the application of phase space finite elements to the first-order transport equation is to result in a production level code, it is

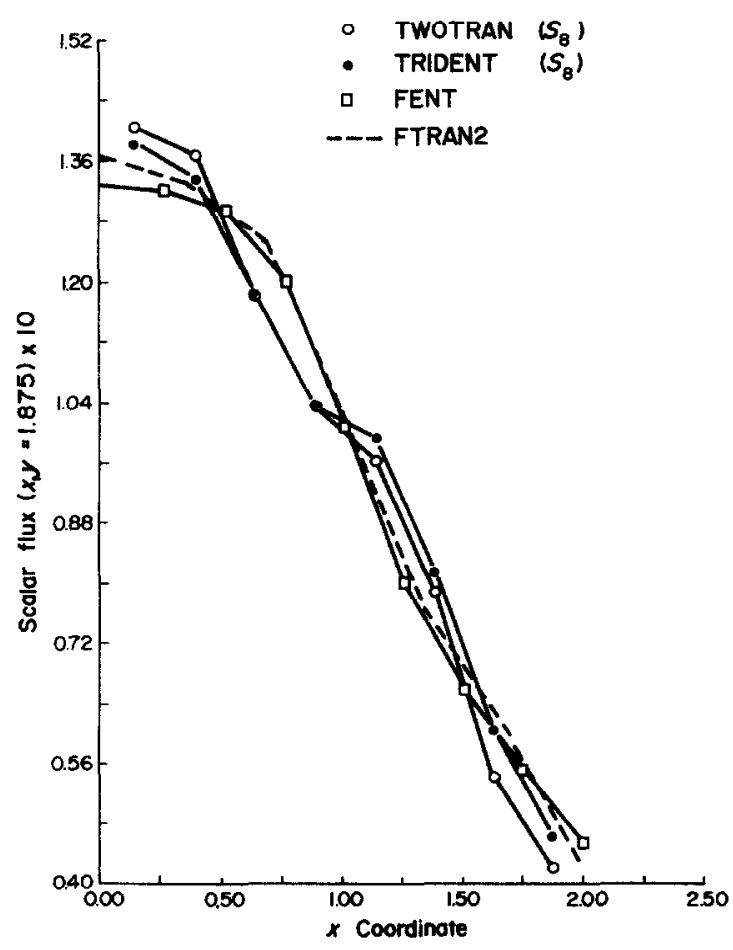

Fig. 4. Scalar flux profiles for ray-effect problem. $\left(c=\frac{1}{3}\right)$ necessary to abandon the block LU-decomposition method in favor of a more efficient numerical method.

\section{SEGMENTATION SOLUTION METHOD}

\subsection{Description of the algorithm}

One promising numerical approach is a segmented technique similar to the interface current methods used to obtain integral transport solutions. Segmentation entails the division of the spatial domain into a coarse mesh. Upon a fine mesh contained in each coarse-mesh segment, the standard Galerkin finite element method is employed. A series of small matrix equations results :

$$
\sum_{j=1}^{N^{k}} A_{i j}^{k} \phi_{j}^{k}=S_{i}^{k} \quad \begin{aligned}
& i=1,2, \ldots, N^{k} \\
& k=1,2, \ldots, M
\end{aligned}
$$

where $M$ is the number of coarse-mesh segments and $N^{k}$ is the number of fine-mesh nodes in the $k$ th segment.

The interface angular fluxes couple adjoining segments by serving as natural incoming boundary conditions. They are included in the source vector $S_{i}^{k}$. The algorithm proceeds by repeated solution of equation (44) until convergence is achieved, beginning with estimates for the unknown interface fluxes. Convergence can be expedited by updating, in a Gauss-Seidel fashion, the terms of the source vectors containing the coupling flux to make maximum use of the most recent results.

\subsection{Efficiency of the segmented algorithm}

The set of coefficient matrices $A^{k}, k=1, \ldots, M$ are LU-factored during the initial global iteration. Their decomposed forms are stored on disk for convenient retrieval during the subsequent iterations for use in back-solves. The number of operations required to factor the set of coefficient matrices is much less than the computational cost of decomposition for the single matrix defined on the entire domain. For example, the matrix of coefficients constructed from rectangular elements on a phase-space domain divided into $X^{2}$ spatial nodes and $U^{2}$ angular nodes requires $X^{4} U^{6}$ operations for factorization. However $S^{2}$ segments with $(X / S)^{2}$ spatial nodes will only require $S^{2}(X / S)^{4} U^{6}$ operations for factorization. Similar reduction in the number of back-solve operations and the storage requirements result from segmentation.

Efficency will also be greatly improved if, as is often the case, only a few of the segment coefficient matrices are unique. This will occur if segments are located in regions of identical material properties, possess the same fine-mesh structure, and have the same type of boundary conditions (reflecting or non-reflecting).

Like a discontinuity in the finite element subspace, a 
segment interface represents a decoupling of the interaction between neighboring elements through the construction of double nodes at the juncture. Spatial discontinuities are useful in representing the angular flux at regions of material discontinuity, as discussed in Section 2.4 for the 1-D case. However, they increase the number of unknowns, and the efficiency of the segmented algorithm will be somewhat offset by this increased cost.

\subsection{Convergence of the segmented algorithm}

The approximate integral law for the segmented algorithm assumed the form (the subspace parameter $h$ is suppressed):

$$
\begin{aligned}
& -(Q, \mathbf{\Omega} \cdot \nabla \psi)+(K Q, \varphi)+\langle Q, \psi\rangle_{\Gamma+} \\
& \quad+\sum_{i, j=1}^{M}\left\langle Q_{i}, \psi_{i}\right\rangle_{\Gamma_{i j}^{+}}-\sum_{i, j=1}^{M}\left\langle Q_{j}, \psi_{i}\right\rangle_{\Gamma_{i j}}=(s, \psi)
\end{aligned}
$$

where

$$
Q, \psi \in S^{h} \in H_{\mathbf{E}}
$$

and

$$
\begin{aligned}
& Q_{i}=Q\left(R_{i} \times \hat{\Omega}\right) \\
& \psi_{i}=\psi\left(R_{i} \times \hat{\Omega}\right)
\end{aligned}
$$

i.e., $Q_{i}$ and $\psi_{i}$ are confined to segmental support

$\Gamma_{i j}^{+}=$out-going boundary of $i$ th segment at $i-j$ interface

$\Gamma_{i j}^{-}=$in-coming boundary of $i$ th segment at $i-j$ interface.

The last two terms on the l.h.s. of equation (45) result from performing the global integration by parts on the streaming term across segment interfaces.

The iteration process is represented by a series of $\left\{Q^{1}, Q^{2}, \ldots, Q^{n}, \ldots\right\}$ that satisfy the $M$ equations:

$$
\begin{aligned}
-\left(Q^{n+1}, \Omega \cdot \nabla \psi\right)+\left(K Q^{n+1}, \psi\right)+\left\langle Q^{n+1}, \psi\right\rangle_{\Gamma_{+}} \\
=\sum_{i>j}\left\langle Q_{j}^{n+1}, \psi_{i}\right\rangle_{\Gamma_{\bar{j}}}+\sum_{i<j}\left\langle Q_{j}^{n}, \psi_{i}\right\rangle_{\Gamma_{\overline{i j}}}+(S, \psi)
\end{aligned}
$$

If $Q^{n+1}$ is sequentially computed as

$$
Q_{1}^{n+1}, Q_{2}^{n+1}, \ldots, Q_{M}^{n+1}
$$

then each equation of equation (46) is the same type as equation (10), for which a unique solution is assured. Hence each $Q^{n+1}$ is uniquely obtained.

Preliminary work by Luskin (1980) has indicated that the series $Q^{n+1}$ will converge. An expression for the convergence rate of segmented phase-space finite elements has not yet been developed.

\subsection{Computational results}

The convergence properties of this algorithm were first explored by Lorence and Martin (1979) in the 1-D four rcgion problem of Reed (1971). Results in cxcellent agreement with FTRAN calculations were obtained in a moderate number of iterations, even with tight pointwise convergence criteria on the angular flux. Even more important for the utility of this algorithm, it was found that the accuracy of the converged result was independent of the chosen starting value for the interface angular fluxes.

A two-dimensional test of the segmented approach was performed on the domain of Fig. 3. A code, SEGMENT2, was developed using rectangular segments and rectangular finite elements. Satisfactory results showing the elimination of the ray effect were obtained. As expected, the computational costs for SEGMENT2 were substantially less than FTRAN2. The algorithm was still somewhat more costly than other transport codes to implement. For instance, the relative costs of the four codes

\section{FTRAN2/SEGMENT2/TRIDENT/FENT}

scaled at $40: 7: 3: 1$. The TRIDENT case was run with the more expensive ray-effect mitigation option that transformed the discrete ordinates equations into spherical harmonic equations. Except for FENT, the four codes solved for the angular flux. The computational cost of SEGMENT2 were not unduly exorbitant and it may be possible to further increase its efficiency.

The memory requirements of SEGMENT2 were also much less than those for FTRAN2. Indeed, if the number of unique segments characterizing a given domain is constant, the memory storage required remains fixed as the mesh is refined. (Actually some additional memory is required to store the larger number of segment solution vectors, but this increase is minor.) While large problems are not constrained by memory allocation, they are computationally limited by the large number of back-solves required. One way of improving the efficiency of the back-solve operations is to implement the code on a vector processor machine.

\section{TIME-DEPENDENT TRANSPORT PROBLEMS}

The finite element method in $x-\mu$ phase-space can be combined with a Crank-Nicholson timedifferencing scheme to solve the time-dependent transport equation. The application of the finite element method is quite similar to that for timeindependent problems. The Galerkin integral law is applied to the first-order transport equation using 
time-dependent expansion coefficients. General boundary conditions (vacuum, reflecting, or specified incoming flux) are included as natural boundary conditions. Applying the Crank-Nicholson scheme to the resulting differential equations yields the algebraic system

$$
\begin{aligned}
\left(\mathbb{M}+\frac{v \Delta t}{2} \mathbb{K}\right) Q^{(n+1)}=(\mathbb{M} & \left.-\frac{v \Delta t}{2} \mathbb{K}\right) \phi^{(n)} \\
& +\frac{v \Delta t}{2}\left(F^{(n+1)}+F^{(n)}\right.
\end{aligned}
$$

where $\phi^{(n)}$ is the angular flux at time $t_{n}, \Delta t$ is the timestep $\left(t_{n}=n \Delta t\right), v$ is the speed, and $F^{(n)}$ is the known source term due to any incoming fluxes or volumetric sources at time $t_{n}$. Conveniently the matrix $\mathbb{K}$ is identical to the matrix of coefficients for the steady-state case. This matrix equation is easily solved by initially factoring $\mathbb{M}+v \Delta t / 2 \mathbb{K}$ into its $L U$ decomposition and then backsolving for $\phi^{(n+1)}$ at each time step.

This method has been incorporated by Martin(1976) into the code FTRAN and applied to a variety of timedependent transport problems in 1-D plane geometries. Detailed comparisons were made with the 1-D timedependent code TIMEX (Hill and Reed, 1976) which uses a finite element treatment of the spatial variable and a discrete ordinates treatment of angle. The range of problems studied included neutron pulse propagation, various incoming source options, and decaying flux problems both in the presence and absence of scattering and absorption. Run times were found to be comparable and numerical agreement was quite good.

It has been well established that the phase-space finite element methods do not suffer from the ray effects that plague discrete orginates methods. This advantage carries over to the time-dependent problems (even in 1-D) since discrete ordinates codes such as TIMEX exhibit a 'time-dependent ray effect'. To illustrate, we consider the propagation in a void of a gaussian shaped pulse for which the initial angular distribution is isotropic in the forward $(\mu>0)$ directions and zero in the backward $(\mu<0)$ directions. A discrete ordinates code will propagate the individual angular fluxes along the $x$-axis with the correct velocities $\mu v$. However, this propagation of the discrete angular modes with different velocities results in their spatial separation with time and consequently leads to a totally fallacious scalar flux, which is illustrated in Fig. 5 (Mehlhorn, 1977).

FTRAN avoids this difficulty since the finite element angular nodes are coupled, even in the absence of scattering. The inherent flexibility in choosing the angular flux mesh in FTRAN leads to a further mitigation of this 'ray' effect for the same number of discrete directions by allowing the user to place more nodes in the direction of propagation. This advantage of the finite element methods in supressing the timedependent ray effect can be clearly seen in Fig. 5 . We conclude that the phase-space finite element method presents an attractive approach to the solution of the time-dependent transport equations.



Fig. 5. Scalar flux profiles for time-dependent problem. 


\section{CONCLUSIONS}

The successful application of the finite element method to the first order neutron transport equation has been demonstrated for 1-D and 2-D geometries. While not competitive with conventional methods for solving the transport equation, such as the highly optimized production level discrete ordinates codes, the finite element method has proven to be feasible and does offer some ad vantages, including the alleviation of the ray effect in multidimensional geometries. The major disadvantage of the finite element method involves the need to solve a large matrix equation directly. This is mitigated by the segmented algorithm described in this paper. This method allows the direct solution of smaller pieces of the matrix equation. This effort is continuing, and we expect further improvement in computing efficiency as this work progresses.

Another area deserving of further attention involves time-dependent transport applications. Our preliminary results indicate a considerable advantage of phase-space finite element methods over discrete ordinate-finite element methods with respect to the 'time-dependent ray effect', which may be observed in 1-D geometries.

There are important advantages and disadvantages that should be considered in weighing the relative merits of applying phase-space finite element methods to the first- or second-order form of the transport equation. The principal advantages of the second-order approach include the reduction of the problem domain due to symmetry and the positive-definite, symmetrical matrix that results due to the self-adjointness of the second-order equation. This latter advantage allows one to utilize iterative methods to solve the resultant matrix equation. However, the solution of the firstorder equation does have some advantages. First, it provides the angular flux as a solution, which cannot be obtained from the even-parity flux alone. (If the oddparity equation is solved, then this advantage disappears.) The treatment of anisotropic scattering to any order is a minor concern with the first-order method. It merely changes the matrix elements, the calculation of which takes a negligible amount of time. This is not true for the second-order equation, where inclusion of anisotropic scattering is a difficult extension from isotropic scattering.
Overall, we consider that first conclusions concerning first-order vs second-order methods are premature, particularly when one notes the significant amount of effort that is underway to develop further each of these methods. However, it can be concluded that neither method compares favorably at the present with discrete ordinates methods except in those situations in which the ray effect is significant and must be eliminated. Hence it is important to continue research into both first- and second-order methods, seeking to make them more competitive with discrete ordinate methods for production code applications.

\section{REFERENCES}

Briggs L. L., Miller W. F. and Lewis E. E. (1975) Nucl. Sci. Engng 57, 205.

Hill T. R. and Reed W. H. (1976) TIMEX : a time-dependent explicit discrete ordinates program for the solution of multigroup transport equations with delayed neutrons. Report LA-6201-MS, Los Alamos Scientific Laboratory.

Lathrop K. D. and Brinkley F. W. (1973) TWOTRAN-II : An interfaced exportable version of the TWOTRAN code for two-dimensional transport. Report LA-4848-MS, Los Alamos Scientific Laboratory.

Lorence L. and Martin W. R. (1979) Trans Am. nucl. Soc. 32, 305.

Luskin M. (1980) Private communication.

Martin W. R. (1975) Trans Am. mucl. Soc. 22, 251.

Martin W. R. (1976) The application of the finite element method to the neutron transport equation. Ph.D. dissertation, The University of Michigan, Ann Arbor, Michigan.

Martin W. R. and Duderstadt J. J. (1977) Nucl. Sci. Engng 62, 371.

Mehlhorn T. A. (1977) Nuclear Engineering Department Report, University of Michigan, Ann Arbor, Michigan.

Miller W. F., Lewis E. E. and Rossow E. C. (1973) Nucl. Sci. Engng 52, 12.

Reed W. H. (1971) Nucl. Sci. Engng 46, 309.

Seed T. J., Miller W. F. and Brinkley F. W.(1977) TRIDENT: a two-dimensional multigroup, triangular mesh discrete ordinates, explicit neutron code. Report LA-6735-MS, Los Alamos Scientific Laboratory.

Strang G. and Fix G.J.(1973) An Analysis of the Finite Element Method, Chapter 6. Prentice-Hall, Englewood Cliffs, New Jersey.

Ukai S. (1972) J. nucl. Sci. Technol. 9, 366.

Yehnert C. E. (1978) Finite element solution of the twodimensional neutron transport equation. Ph.D. Dissertation, The University of Michigan, Ann Arbor, Michigan. 